\title{
Zinc and melatonin supplementation ameliorates brain cortex tissue damage in DMBA-induced breast cancer in rats
}

\author{
Gulbahce Mutlu E ${ }^{1}$, Baltaci SB ${ }^{2}$ \\ KTO Karatay University, Medical Faculty, Department of Physiology, Konya, Turkey. \\ saltukbugrabaltaci@selcuk.edu.tr
}

\section{ABSTRACT}

BACKGROUND: This study aims to investigate the effects of zinc and melatonin supplementation on lipid peroxidation in the brain cortex in DMBA-induced breast cancer in female rats.

METHODS: A total of 42 recently weaned Wistar rats were divided into 5 groups as follows: Control (Group 1), DMBA Control (Group 2), DMBA+Zinc (Group 3), DMBA+Melatonin (Group 4), DMBA+Melatonin \& Zinc

(Group 5). At the end of the study, all animals were sacrificed by decapitation and Malondialdehyde (MDA) and glutathione (GSH) levels in brain cortex tissue samples were determined via spectrophotometric methods. RESULTS: The highest MDA levels were in the DMBA-treated group (Group 2) $(p<0.05)$. MDA levels in Group 3, Group 4, and Group 5 were significantly lower than in group $2(p<0.05)$. Also, GSH levels in group 3,4 , and 5 were significantly higher than in group $2(p<0.05)$.

CONCLUSION: There are no reports on whether DMBA-induced experimental breast cancer affects oxidative stress in brain tissue. In this respect, our study revealed that the increased brain cortex tissue damage in DMBA-induced breast cancer is alleviated by Zinc, melatonin, or combined zinc and melatonin treatment (Fig. 3, Ref. 26). Text in PDF www.elis.sk

KEY WORDS: DMBA, breast cancer, brain tissue, MDA, GSH, zinc, melatonin.

\section{Introduction}

Human breast cancer is an extremely complex and dangerous disease (1). Cancer treatment has become increasingly challenging and incurable due to the emergence and prevalence of multidrug resistance, the non-specific systemic distribution of anti-tumor agents, insufficient drug concentrations reaching the tumor site, and the limited ability to monitor cytotoxicity and therapeutic responses $(1,2)$. In order to overcome this problem, new strategies are investigated to treat cancer or help the treatment currently being carried out. Due to its similarity to human breast cancer, the experimental breast cancer model created in rats is a highly preferred method (3). DMBA (7,12-Dimethylbenz(a)anthracene) induced breast carcinomas have become the standard laboratory model of breast carcinogenesis in female rats (4). DMBA is a synthetic polycyclic aromatic hydrocarbon commonly used as a prototype agent in mutation and cancer research since DMBAmediated biochemical, molecular, genetic and histopathological changes in rats are similar to those observed in human cancers (5).

${ }^{1}$ KTO Karatay University, Medical Faculty, Department of Physiology, Konya, Turkey, and ${ }^{2}$ Selcuk University, Medical Faculty, Department of Physiology, Konya, Turkey

Address for correspondence: Saltuk Bugra Baltaci, Selcuk University, Medical Faculty, Department of Physiology, 42031, Konya, Turkey.

Acknowledgements: This study was supported by Selcuk University, Scientific Research Projects Coordination Unit.
Studies have shown the role of trace elements in anticancer mechanisms (6). Zinc, as a catalytic component of more than 300 enzymes, especially those involved in the antioxidant defense system, such as metallotionins, copper/zinc superoxide dismutase, is a trace element that attracts a lot of attention in anti-carcinogenic events (6). Zinc also functions as the cofactor of proteins that control responses to matrix metalloproteinases (MMPs), which are involved in the pathogenesis of breast cancer (7). Changes in zinc concentrations may play an important role in cell dysfunction and proliferation, including the development and progression of this disease (8).

The role of melatonin produced and secreted by the pineal gland, as an anti-carcinogenic agent has been extensively investigated in the past few decades (9). Melatonin has been evaluated in various types of cancer, especially breast carcinoma (10). While melatonin inhibits tumor growth by reducing cell proliferation, it also has anti-metastatic, pro-apoptotic, anti-angiogenic, antioxidant/anti-mutagenic and immuno-enhancing effects $(9,10)$. These effects of melatonin make it an excellent agent to reduce the risk of carcinogenesis in humans $(9,10)$.

The aim of this study is to investigate the effects of zinc and melatonin administration on lipid peroxidation in brain tissue (cortex) in DMBA-induced breast cancer in female rats.

\section{Methods}

\section{Animal material and groups}

The study included recently weaned (40-day old) female rats of Wistar type. The study protocol was approved by Selcuk Uni- 
$749-752$

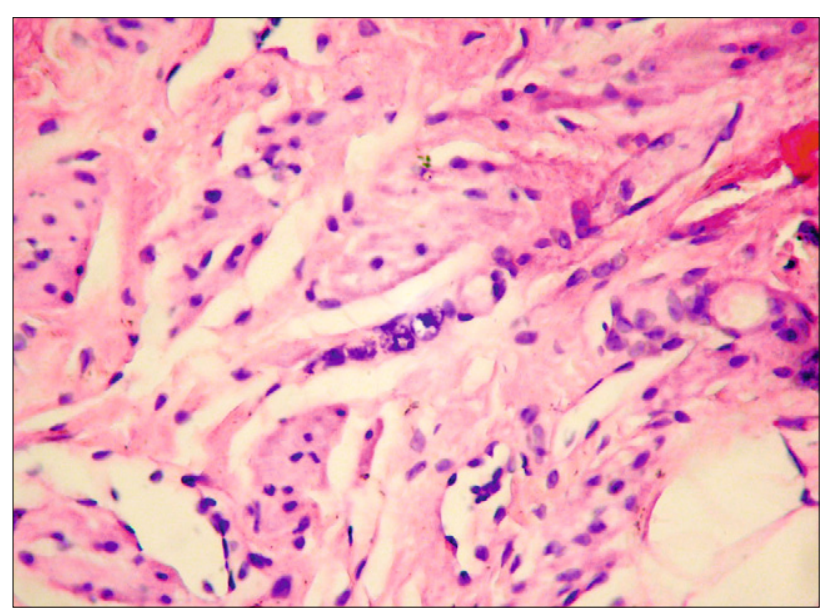

Fig. 1. Malignant changes were determined in breast tissue (HE: X40). a, b, c: Intergroup means with different superscripts in the same column show significant difference $(p<0.05)$.

versity Experimental Medicine Research and Application Center, Animal Experiment Ethics Committee (2016-31). The 42 female rats included in the study were grouped as follows:

Group 1 (n:6), Control Group: The group was fed on a normal diet and not subjected to any procedure.

Group 2 (n:6), DMBA Control Group: The animals in this group were administered $80 \mathrm{mg} / \mathrm{kg}$ 7,12-dimethyl[a]anthracene (DMBA) in colza oil (canola) through gavage to induce a tumor and fed on a normal diet.

Group 3 (n:10), DMBA+Zinc Group: The animals in this group were administered $80 \mathrm{mg} / \mathrm{kg}$ 7,12-dimethyl[a]anthracene (DMBA) in colza oil (canola) through gavage to induce a tumor, and supplemented with $5 \mathrm{mg} / \mathrm{kg} /$ day intraperitoneal (i.p.) zinc along with their normal diet for 4 weeks.

Group 4 (n:10) DMBA+Melatonin Group: The animals in this group were administered $80 \mathrm{mg} / \mathrm{kg}$ 7,12-dimethyl[a]anthracene (DMBA) in colza oil (canola) through gavage to induce a tumor, and supplemented with $5 \mathrm{mg} / \mathrm{kg} /$ day intraperitoneal (i.p.) melatonin along with their normal diet for 4 weeks.

Group 5 (n:10) DMBA+Melatonin and Zinc Group: The animals in this group were administered $80 \mathrm{mg} / \mathrm{kg}$ 7,12-dimethyl[a] anthracene (DMBA) in colza oil (canola) through gavage to induce a tumor, and supplemented with $5 \mathrm{mg} / \mathrm{kg}$ /day intraperitoneal (i.p.) melatonin and zinc combination along with their normal diet for 4 weeks.

\section{Feeding experimental animals}

The experimental animals were housed in a separate room at the Selcuk University Experimental Medicine Research and Application Centeruntil until the end of the study. They were kept in an environment with standard temperature and light $\left(21 \pm 1^{\circ} \mathrm{C}\right.$ and 12-hour dark, 12-hour light). Rats were fed with tap water and rat feed provided by Selcuk University Experimental Medicine Research and Application Center. They were accommodated in special cages which were cleaned daily. The animals were given 10 grams of feed per 100 grams of body weight.

\section{Experimental procedures}

Induction of breast cancer

To induce breast cancer, 7,12-dimethylbenz[a]anthracene (DMBA) supplied by Sigma-Aldrich company (St. Louis, MO, the USA) was used. For this purpose, a single dose of $80 \mathrm{mg} / \mathrm{kg}$ dimethylbenz[a]anthracene (DMBA) in colza oil (canola) was administered through gavage. One week after the administration, the animals' breast tissues were examined by palpation to check the enlargement of breast tissue. After the enlargement in the breast tissue became evident, 6 rats from among the 36 rats which were administered DMBA were randomly chosen and their breast tissue samples were collected under general anesthesia. After the presence of the tumor was pathologically detected by light microscopic examination, zinc and melatonin supplementation started (Fig. 1). Tumor development was pathologically detected in the 10th week after DMBA administration.

\section{Tissue sample collection}

After 4-week treatments, brain cortex tissue samples were obtained from animals sacrificed under general anesthesia. Tissue samples were kept at $-80^{\circ} \mathrm{C}$ until the time of analysis.

\section{Biochemical analyses}

Identification of Brain Cortex Tissue Malondialdehyde (MDA) Analysis

MDA analyses in the brain cortex tissue were carried out according to TBA (thiobarbituric acid) technique developed by Uchiyama and Miharama (11). Results of tissue MDA analyses were determined as $\mathrm{nmol} / \mathrm{g}$ tissue in the spectrophotometer.

Identification of Brain Cortex Tissue Glutathione Analysis

GSH in the brain cortex tissue was analyzed using the Biuret method as described by Ellman (12) and the results were determined as $\mathrm{mg} / \mathrm{g}$ tissue.

\section{Statistical analysis}

Statistical evaluation of the results was made with SPSS 22.0 statistical software, and arithmetic means and standard deviations of all parameters were calculated. Kruskal-Wallis $\mathrm{H}$ test was used to determine the differences between the groups, and Mann-

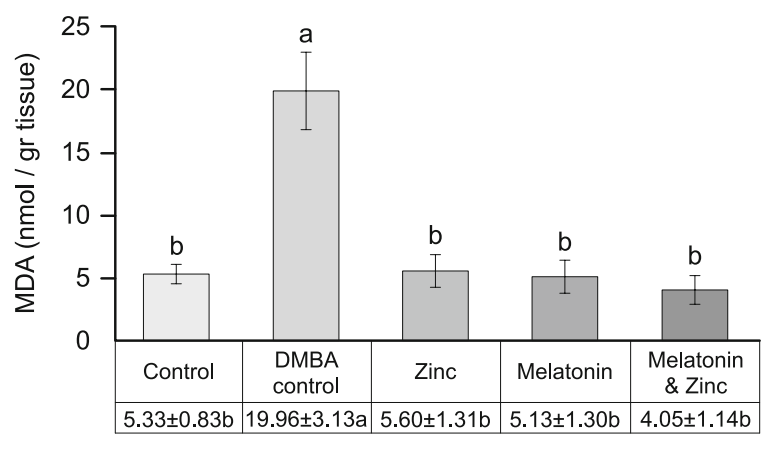

Fig. 2. Comparison of MDA Levels in Cortex Tissue of the Groups. a, $b, c$ : Intergroup means with different superscripts in the same column show significant difference $(\mathbf{p}<\mathbf{0 . 0 5})$. 


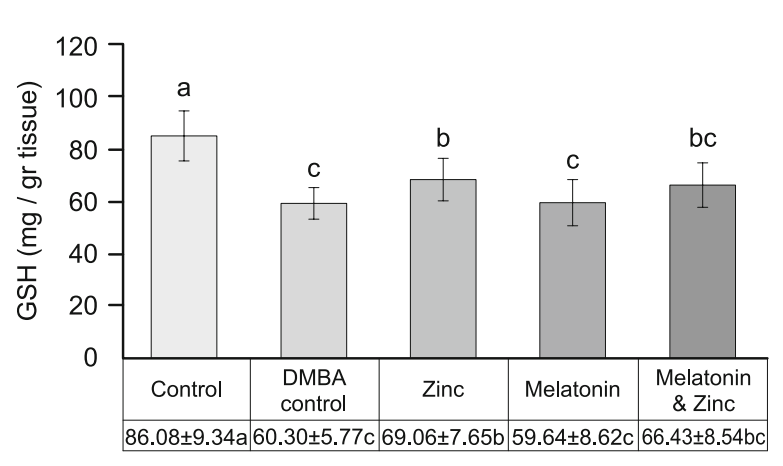

Fig. 3. Comparison of GSH Levels in Cortex Tissue of the Groups.

-Whitney U test was utilized to find the group causing difference. $\mathrm{p}<0.05$ values were considered statistically significant.

\section{Results}

The highest brain MDA levels were found in the control group (G2) that developed DMBA-induced breast cancer $(p<0.05)$. MDA levels of DMBA + Zinc (G3) group, DMBA + Melatonin (G4) group and DMBA (G5) group where Melatonin and Zinc were applied in combination were significantly lower than in G2 $(\mathrm{p}<0.05)$ (Fig. 2). Similarly, the study revealed that the brain GSH levels of DMBA + Zinc (G3) group, DMBA + Melatonin (G4) group, and DMBA group (G5) where Melatonin and Zinc were administered in combination were significantly higher than in the control group (G2) with DMBA-induced breast cancer in $(\mathrm{p}<0.05)$ (Fig. 3).

\section{Discussion}

DMBA, which is used to induce experimental breast carcinomas in rats, affects various biochemical and physiopathological processes and causes oxidative damage by disrupting tissue redox balance (13). As a result, DMBA increases the formation of reactive oxygen species (ROS) and causes oxidative stress (14). Increased oxidative stress plays a role in carcinogenesis (15). Central nervous system metastasis has been reported in $15-25 \%$ of breast cancer patients and incidence rates are increasing (16). However, there are no reports on how DMBA-induced experimental breast cancer affects oxidative stress in brain tissue. In our study, the highest MDA levels in the brain cortex were found in the control group (G2) with DMBA-induced breast cancer. This is a critical finding, as it shows increased tissue damage in the brain cortex in DMBA-induced rats.

Many studies show that zinc deficiency is highly associated with increased levels of oxidative stress biomarkers such as lipid peroxidation products and DNA oxidation products $(17,18)$. Zinc supplement suppresses or alleviates these negative effects (17, 18). These findings suggest that zinc may have a protective role as a pro-antioxidant agent or agent in reducing the production and accumulation of free radical products. Indeed, zinc acts as a common factor for important enzymes that contribute to the proper functioning of the antioxidant defense system (18). In addition, this mineral protects against oxidative damage and induces metallothionein synthesis as it affects the stabilization of cell membranes (18). Metallothionein plays a role in reducing hydroxyl radicals $(\mathrm{OH})$ and eliminating reactive oxygen species produced under stress conditions (18). Studies have highlighted the role of glutathione in the regulation of peroxidase and metallothionein expression, as well as the role of zinc as a co-factor for superoxide dismutase $(17,18)$. In addition, zinc competes with iron and copper in the cell membrane, inhibits the NADPH-oxidase enzyme, and reduces chronic inflammation and hyperglycemia $(18,19)$. The zinc supplement given to animals with DMBAinduced breast cancer resulted in significant suppression in MDA levels in brain cortex tissue in our study. This finding is consistent with the findings of the researchers who emphasize that zinc has a protective effect against oxidative damage $(17,18)$. It was pointed out that zinc supplementation may prevent tissue damage in the early stages of cancer, but this preventive effect may be dose-dependent (20). The report of Onaolapo et al (21), showing that zinc supplementation prevents oxidative stress in the brain tissues of mice, is compatible with decreased MDA in the brain cortex, which we found in our study with zinc supplementation. In our study, zinc supplementation to animals with DMBA-induced breast cancer led to a significant increase in GSH levels in brain cortex tissue compared to the DMBA group. MDA values, which we found to be suppressed in the brain cortex tissue with zinc supplementation, are the result of an increase in GSH levels thanks to zinc supplementation. Zinc has been found to be effective in preventing tissue damage by increasing antioxidatnt activity in breast cancer treatment (22). As a matter of fact, antioxidant activity also has an important role in the basis of the anti-cancer effects of zinc. In this respect, the increased GSH and suppressed MDA levels we found in the DMBA group with zinc supplementation were compatible with the reports presented.

Melatonin, a pineal hormone, has strong anti-breast cancer activity and aging-related decrease in melatonin levels is associated with tumoral events in the body (23). The direct free radical scavenging effect of the hormone melatonin has been known for a long time $(23,24)$. In addition, melatonin is a highly effective internal antioxidant that regulates glutathione synthesis and stimulates antioxidative enzymes, including glutathione peroxidase and glutathione reductase (24). The antioxidant functions of melatonin mentioned make this molecule an important endogenous actor that prevents free radical damage $(23,24)$. In this study, melatonin supplementation in animals with DMBA-induced breast cancer resulted in significant suppression of MDA levels in brain cortex tissue and a significant increase in GSH levels. Abadi et al (25) reported that melatonin supplementation in mice exposed to radiation suppresses tissue and plasma MDA levels, resulting in a significant increase in GSH levels in tumoral tissues when compared to non-melatonin supplemented rats. Kurhaluk et al (26) reported that increased oxidative damage in both breast and heart tissue was prevented by supplementing melatonin in breast cancer-induced female rats. Our findings are in parallel with the findings of the researchers whose reports are presented above. 
In our study, we obtained similar results with the combined administration of zinc and melatonin in rats with DMBA-induced breast cancer. That is, the combined administration of melatonin and zinc suppressed the oxidative damage in the brain tissue by increasing the antioxidant activity in rats with breast cancer. However, it should be emphasized that the combined administration of zinc and melatonin was not more successful than supplementation of zinc or melatonin alone.

There are no reports of how DMBA-induced experimental breast cancer affects oxidative stress in brain tissue. In this respect, our study revealed that; 1 . Oxidative damage occurs in the brain tissue cortex of rats with DMBA-induced breast cancer. 2. Tissue damage in the brain cortex is prevented with zinc, melatonin and zinc + melatonin combination. 3. The preventive effect of zinc + melatonin combination on oxidative damage was not different from the administration of zinc or melatonin alone. The absence of a difference may be related to the duration or dose of the zinc+melatonin combination administered.

The findings of the study showed that increased brain tissue (cortex) damage in DMBA-induced breast cancer is suppressed by zinc, melatonin and zinc+melatonin supplementation. Supplementation of zinc, melatonin and zinc+melatonin combination may contribute to the prevention of tissue damage in tumoral events.

\section{References}

1. Sofi MS, Sateesh MK, Bashir M, Ganie MA, Nabi S. Chemopreventive and anti-breast cancer activity of compounds isolated from leaves of Abrusprecatorius L.3 Biotech 2018; 8 (8): 371.

2. Misra R, Acharya S, Sahoo SK. Cancer nanotechnology: application of nanotechnology in cancer therapy.Drug Discov Today 2010; 15 (19-20): 842-850.

3. Samy RP, Gopalakrishnakone P, Ignacimuthu S. Anti-tumor promoting potential of luteolin against 7,12-dimethylbenz (a)anthracene-induced mammary tumors in rats.Chem Biol Interact 2006; 164 (1-2): 1-14.

4. Russo J, Russo IH. Experimentally induced mammary tumors in rats. Breast Cancer Res Treat. 1996; 39 (1): 7-20.

5. Izzotti A, Camoirano A, Cartiglia C, Grubbs CJ, Lubet RA, Kelloff GJ, Flora SD. Patterns of DNA adduct formation in liver and mammary epithelial cells of rats treated with 7, 12-dimethylbenz (a)anthracene, and selective effects of chemopreventive agents. Can Res 1999; 59: 4285-4290.

6. Kumar R, Razab S, Prabhu K, Ray S, Prakash B. Serum butyrylcholinesterase and zinc in breast cancer.J Cancer Res Ther 2017; 13 (2): 367-370.

7. Cui N, Hu M, Khalil RA. Biochemical and Biological Attributes of Matrix Metalloproteinases. Prog Mol BiolTransl Sci 2017; 147: 1-73.

8. da Cruz RS, Andrade FO, Carioni VMO, Rosim MP, Miranda MLP, Fontelles CC, de Oliveira PV, Barbisan LF, Castro IA, Ong TP. Dietary zinc deficiency or supplementation during gestation increases breast cancer susceptibility in adult female mice offspring following a J-shaped pattern and through distinct mechanisms.Food Chem Toxicol 2019; 134: 110813.

9. Ferreira LC, Orso F, Dettori D, Lacerda JZ, Borin TF, Taverna D, Zuccari DAPC. The role of melatonin on miRNAs modulation in triple-negative breast cancer cells.PLoS One 2020; 15 (2): e0228062.

10. Kubatka P, Zubor P, Busselberg D, Kwon TK, Adamek M, Petrovic D, Opatrilova R, Gazdikova K, Caprnda M, Rodrigo L, Danko J, Kruzliak P. Melatonin and breast cancer: Evidences from preclinical and human studies.Crit Rev Oncol Hematol 2018; 122: 133-143.
11. Mihara M, Uchiyama M. Determination of malonaldehyde precursor in tissues by thiobarbituric acid test.Anal Biochem 1978; 86 (1): 271-278.

12. Sedlak J, Lindsay RH. Estimation of total, protein-bound, and nonprotein sulfhydryl groups in tissue with Ellman's reagent.Anal Biochem 1968; 25 (1): 192-205.

13. Batcioglu K, Uyumlu AB, Satilmis B, Yildirim B, Yucel N, Demirtas H, Onkal R, Guzel RM, Djamgoz MB. Oxidative stress in the in vivo DMBA rat model of breast cancer: suppression by a voltage-gated sodium channel inhibitor (RS100642). Basic Clin PharmacolToxicol 2012; 111 (2): $137-41$.

14. Zeweil MM, Sadek KM, Taha NM, El-Sayed Y, Menshawy S. Graviola attenuates DMBA-induced breast cancer possibly through augmenting apoptosis and antioxidant pathway and downregulating estrogen receptors. Environ Sci Pollut Res Int. 2019; 26 (15): 15209-15217.

15. Karnam KC, Ellutla M, Bodduluru LN, Kasala ER, Uppulapu SK, Kalyankumarraju M, Lahkar M. Preventive effect of berberine against DMBA induced breast cancer in female Sprague Dawley rats. Biomed Pharmacother 2017; 92: 207-214.

16. Custódio-Santos T, Videira M, Brito MA. Brain metastasization of breast cancer. BiochimBiophys Acta Rev Cancer 2017; 1868 (1): 132-147.

17. Prasad AS, Bao B. Molecular Mechanisms of Zinc as a Pro-Antioxidant Mediator: Clinical Therapeutic Implications. Antioxidants (Basel) 2019; 8 (6): pii: E164.

18. Marreiro DD, Cruz KJ, Morais JB, Beserra JB, Severo JS, de Oliveira AR. Zinc and Oxidative Stress: Current Mechanisms. Antioxidants (Basel) 2017; 6 (2): pii: E24.

19. Cruz KJ, de Oliveira AR, Marreiro Ddo N. Antioxidant role of zinc in diabetes mellitus. World J Diabetes 2015; 6 (2): 333-337.

20. Bobrowska-Korczak B, Skrajnowska D, Kiss AK, Wrzesien R, Bielecki W, Orzoł A, Zebrowski P, Bialek S. Lipid peroxidation as a predictive biomarker of the early stage of cancer. J BiolRegulHomeost Agents 2019; 33 (3): 799-810.

21. Onaolapo OJ, Jegede OR, Adegoke O, Ayinde MO, Akeredolu OM, Onaolapo AY. Dietary zinc supplement militates against ketamineinduced behaviours by age-dependent modulation of oxidative stress and acetylcholinesterase activity in mice. Pharmacol Rep 2020; 72 (1): 55-66.

22. Famurewa AC, Ekeleme-Egedigwe CA, David EE, Eleazu CO, Folawiyo AM, Obasi NA. Zinc abrogates anticancer drug tamoxifen-induced hepatotoxicity by suppressing redox imbalance, NO/iNOS/NF-kBsignaling, and caspase-3-dependent apoptosis in female rats. Toxicol Mech Methods 2020; 30 (2): 115-123.

23. Hill SM, Cheng C, Yuan L, Mao L, Jockers R, Dauchy B, Blask DE. Age-related decline in melatonin and its MT1 receptor are associated with decreased sensitivity to melatonin and enhanced mammary tumor growth. Curr Aging Sci 2013; 6 (1): 125-133.

24. Reiter RJ, Mayo JC, Tan DX, Sainz RM, Alatorre-Jimenez M, Qin L. Melatonin as an antioxidant: under promises but over delivers. J Pineal Res 2016; 61 (3): 253-278.

25. Abadi SHMH, Shirazi A, Alizadeh AM, Changizi V, Najafi M, Khalighfard S, Nosrati H. The effect of melatonin on superoxide dismutase and glutathione peroxidase activity, and malondialdehyde levels in the targeted and the non-targeted lung and heart tissues after irradiation in xenograft mice colon cancer. Curr Mol Pharmacol 2018; 11 (4): 326-335.

26. Kurhaluk N, Bojkova B, Radkowski M, Zaitseva OV, Kyriienko S, Demkow U, Winklewski PJ. Melatonin and Metformin Diminish Oxidative Stress in Heart Tissue in a Rat Model of High Fat Diet and Mammary Carcinogenesis. Adv Exp Med Biol 2018; 1047: 7-19.

Received April 30, 2020. Accepted May 30, 2020. 